\title{
Absolute Continuity of Fuzzy Measures and Convergence of Sequence of Measurable Functions
}

\author{
Jun $\mathrm{Li}^{1,2}$ \\ 1 School of Sciences, Communication University of China, Beijing 100024, China; lijun@cuc.edu.cn \\ 2 School of Data Science and Media Intelligence, Communication University of China, Beijing 100024, China
}

Received: 31 January 2020; Accepted: 1 May 2020; Published: 5 May 2020

\begin{abstract}
In this note, the convergence of the sum of two convergent sequences of measurable functions is studied by means of two types of absolute continuity of fuzzy measures, i.e., strong absolute continuity of Type I, and Type VI. The discussions of convergence a.e. and convergence in measure are done in the general framework relating to a pair of monotone measures, and general results are shown. The previous related results are generalized.
\end{abstract}

Keywords: fuzzy measure; absolute continuity; convergence in measure; fuzzy integral

\section{Introduction}

In fuzzy measure and fuzzy integral theory, many results in the classical measure theory no longer hold generally without additional conditions for fuzzy measures. For example, for Lebesgue measurable functions sequences $\left\{f_{n}\right\}_{n \in \mathbb{N}}$ and $\left\{g_{n}\right\}_{n \in \mathbb{N}}$, we have

$$
f_{n} \rightarrow f \text { a.e. }[m] \text { and } g_{n} \rightarrow g \text { a.e. }[m] \Longrightarrow f_{n}+g_{n} \rightarrow f+g \text { a.e. }[m]
$$

and

$$
f_{n} \stackrel{m}{\longrightarrow} f \text { and } g_{n} \stackrel{m}{\longrightarrow} g \Longrightarrow f_{n}+g_{n} \stackrel{m}{\longrightarrow} f+g,
$$

where $m$ is the Lebesgue measure and the convergence concerns convergence almost everywhere and convergence in measure [1,2]. However, for a fuzzy measure $\mu$, in general, the above Equations (1) and (2) may not be true.

The above Equations (1) and (2) were generalized to fuzzy measure spaces under the conditions of weak null-additivity and pseudometric generating property of set functions [3].

In this note, we consider a pair of fuzzy measures $\lambda$ and $v$ defined on the same measurable space $(X, \mathcal{A})$. Under the condition of strong absolute continuity of Type I (resp. strong absolute continuity of Type VI), we obtain the following result:

$$
\begin{gathered}
f_{n} \rightarrow f \text { a.e. }[v] \text { and } g_{n} \rightarrow g \text { a.e. }[v] \Longrightarrow f_{n}+g_{n} \rightarrow f+g \text { a.e. }[\lambda] \\
\left.\quad \text { (resp. } f_{n} \stackrel{v}{\longrightarrow} f \text { and } g_{n} \stackrel{v}{\longrightarrow} g \Longrightarrow f_{n}+g_{n} \stackrel{\lambda}{\longrightarrow} f+g\right) .
\end{gathered}
$$

Comparing Equation (3) with Equation (1), and Equation (4) with Equation (2), respectively, we see that the general results are obtained in the framework concerning a pair of monotone measures. The previous related results in [3,4] (see also [5]) are recovered. 


\section{Preliminaries}

Let $(X, \mathcal{A})$ be a measurable space, i.e., $X$ is a nonempty set and $\mathcal{A}$ is a $\sigma$-algebra of subsets of $X$. Let $\mathcal{F}$ denote the collection of all $\mathcal{A}$-measurable functions $f: X \rightarrow[0,1]$ (such a function $f$ is also called a fuzzy set on $X$ ). The symbols " $\vee$ " and " $\wedge$ " denote logical addition and logical multiplication (i.e., for any $a, b \in[0,1], a \vee b=\max \{a, b\}$, and $a \wedge b=\min \{a, b\}$ ), respectively.

\subsection{Fuzzy Measures and Integrals}

A fuzzy measure on $(X, \mathcal{A})$ is a real valued set function $\mu: \mathcal{A} \rightarrow[0,1]$ satisfying the conditions: (FM1) $\mu(\varnothing)=0$ and $\mu(X)>0$; (FM2) $\mu(P) \leq \mu(Q)$ whenever $P \subset Q$ and $P, Q \in \mathcal{A}$ (see [6,7]).

A fuzzy measure is also known as "non-additive measure", "capacity", "monotone measure", "non-additive probability", etc (see [8-11]).

Let $\mathfrak{F M}$ denote the set of all fuzzy measures defined on $(X, \mathcal{A})$. For $\lambda, v \in \mathfrak{F M}$, let $(\lambda, v)$ denote the order pair of $\lambda$ and $v$, i.e., $(\lambda, v) \in \mathfrak{F M} \times \mathfrak{F M}$.

We recall fuzzy integral [6] (it is also called Sugeno integral, see [12,13]).

Let $(\mu, f) \in \mathfrak{F M} \times \mathcal{F}$. The fuzzy integral of $f$ on $X$ with respect to $\mu$, is defined by

$$
\text { (S) } \int f d \mu=\bigvee_{0 \leq \alpha \leq 1}[\alpha \wedge \mu(\{x \in X \mid f(x) \geq \alpha\})] \text {. }
$$

For $A \in \mathcal{A}$, we define (S) $\int_{A} f d \mu=(S) \int f \chi_{A} d \mu$, where $\chi_{A}$ is the characteristic function of $A$. Note that the Sugeno integral is a special kind of nonlinear integral.

We define the conjugate $\bar{\mu}$ of $\mu$ by

$$
\bar{\mu}(A)=\mu(X)-\mu(X \backslash A), \quad A \in \mathcal{A} .
$$

Then $\bar{\mu} \in \mathfrak{F M}$ and $\overline{\bar{\mu}}=\mu$.

\subsection{Convergence of Sequence of Measurable Functions}

Let $\mu \in \mathfrak{F M}$ and $f, f_{n} \in \mathcal{F}(n=1,2, \ldots)$. We say that

(1) $\left\{f_{n}\right\}_{n \in \mathbb{N}}$ converges almost everywhere to $f$ on $X$ with respect to $\mu$, and denote it by $f_{n} \stackrel{\text { a.e. }}{\longrightarrow} f[\mu]$, if there is a subset $E \in \mathcal{A}$ such that $\mu(E)=0$ and $f_{n} \rightarrow f$ on $X \backslash E$;

(2) $\left\{f_{n}\right\}_{n \in \mathbb{N}}$ converges pseudo-almost everywhere to $f$ on $X$ with respect to $\mu$, and denote it by $f_{n} \stackrel{\text { p.a.e. }}{\longrightarrow} f[\mu]$, if there is a subset $F \in \mathcal{A}$ such that $\mu(X \backslash F)=\mu(X)$ and $f_{n} \rightarrow f$ on $X \backslash F$;

(3) $\left\{f_{n}\right\}_{n \in \mathbb{N}}$ converges to $f$ in measure $\mu$ (resp. pseudo-in measure $\mu$ ) on $X$, denoted by $f_{n} \stackrel{\mu}{\longrightarrow} f$ (resp. $\left.f_{n} \stackrel{p \cdot \mu}{\longrightarrow} f\right)$, if for any $\sigma>0, \lim _{n \rightarrow \infty} \mu\left(\left\{x \in X|| f_{n}(x)-f(x) \mid \geq \sigma\right\}\right)=0$ (resp. $\lim _{n \rightarrow+\infty} \mu(\{x \in X \mid$ $\left.\left.\left.\left|f_{n}(x)-f(x)\right|<\sigma\right\}\right)=\mu(X)\right)$.

Proposition 1. Let $\mu \in \mathfrak{F M}$. Then

(1) $f_{n} \stackrel{\text { a.e. }}{\longrightarrow} f[\mu]$ if and only if $f_{n} \stackrel{\text { p.a.e. }}{\longrightarrow} f[\bar{\mu}]$;

(2) $f_{n} \stackrel{\mu .}{\longrightarrow} f$ if and only if $f_{n} \stackrel{p \cdot \bar{\mu}}{\longrightarrow} f$.

\subsection{Absolute Continuity of Fuzzy Measures}

We recall several types of absolute continuity of fuzzy measures.

Definition 1. [14] Let $(\lambda, v) \in \mathfrak{F M} \times \mathfrak{F M . ~ W e ~ s a y ~ t h a t ~}$

(1) $\lambda$ is absolutely continuous of Type I with respect to $v$, denoted by $\lambda \ll_{I} v$, if for any $A \in \mathcal{A}$, we have

$$
\nu(A)=0 \Longrightarrow \lambda(A)=0
$$


(2) $\lambda$ is absolutely continuous of Type VI with respect to $v$, denoted by $\lambda \ll_{V I} v$, if for any $\left\{A_{n}\right\}_{n \in \mathbb{N}} \subset \mathcal{A}$, we have

$$
v\left(A_{n}\right) \rightarrow 0(n \rightarrow \infty) \Longrightarrow \lambda\left(A_{n}\right) \rightarrow 0(n \rightarrow \infty)
$$

Inspired by the concepts of weak null-additivity and pseudometric generating property of set functions we generalized the above two types of absolute continuity, i.e., Type I and Type VI (see [3]). Let us first recall weak null-additivity and pseudometric generating property of fuzzy measures.

Let $\mu \in \mathfrak{F M}$. (1) $\mu$ is called weakly null-additive [11,15], if for any $P, Q \in \mathcal{A}$, we have

$$
\mu(P)=\mu(Q)=0 \Longrightarrow \mu(P \cup Q)=0 .
$$

(2) $\mu$ is called to have pseudometric generating property (briefly, (p.g.p.)) $[4,16]$, if for any sequences $\left\{P_{n}\right\}_{n \in \mathbb{N}} \subset \mathcal{A}$ and $\left\{Q_{n}\right\}_{n \in \mathbb{N}} \subset \mathcal{A}$, we have

$$
\mu\left(P_{n}\right) \vee \mu\left(Q_{n}\right) \rightarrow 0(n \rightarrow \infty) \Longrightarrow \mu\left(P_{n} \cup Q_{n}\right) \rightarrow 0(n \rightarrow \infty) .
$$

Definition 2. (Li et al. [3]) Let $(\lambda, v) \in \mathfrak{F M} \times \mathfrak{F M}$. We say that

(1) $\lambda$ is strongly absolute continuous of Type I with respect to $v$, denoted by $\lambda \ll_{I}^{(s)} v$, if

$$
v(A)=v(B)=0 \Longrightarrow \lambda(A \cup B)=0 ;
$$

(2) $\lambda$ is strongly absolute continuous of Type VI with respect to $v$, denoted by $\lambda \ll_{V I}^{(s)} v$, if for any sequences $\left\{A_{n}\right\}_{n \in \mathbb{N}} \subset \mathcal{A}$ and $\left\{B_{n}\right\}_{n \in \mathbb{N}} \subset \mathcal{A}$, we have

$$
v\left(A_{n}\right) \vee v\left(B_{n}\right) \rightarrow 0(n \rightarrow \infty) \Longrightarrow \lambda\left(A_{n} \cup B_{n}\right) \rightarrow 0(n \rightarrow \infty) .
$$

Note: Comparing Equation (10) with Equation (8), and Equation (11) with Equation (9), respectively, we have (1) $\mu \ll_{I}^{(s)} \mu$ if and only if $\mu$ is weakly null-additive, and (2) $\mu \ll_{V I}^{(s)} \mu$ if and only if $\mu$ has pseudometric generating property.

Proposition 2. Let $(\lambda, v) \in \mathfrak{F M} \times \mathfrak{F M}$.

(1) If $\lambda \ll_{V I} v$, then $\lambda \ll_{I} v$.

(2) If $\lambda \ll_{V I}^{(s)} v$, then $\lambda \ll_{I}^{(s)} v$.

(3) $\lambda \ll_{I}^{(s)} v$ implies $\lambda \ll_{I} v$, and $\lambda \ll_{V I}^{(s)} v$ implies $\lambda \ll_{V I} v$.

The inverse statements in (1), (2) and (3) may not hold.

\section{Main Results}

The following Theorems 1-3 are our main results.

Theorem 1. Let $(\lambda, v) \in \mathfrak{F M} \times \mathfrak{F M}$. Then, we have

(1) for all $f \in \mathcal{F}$ and all $\left\{f_{n}\right\}_{n \in \mathbb{N}} \subset \mathcal{F}$,

$$
f_{n} \stackrel{\text { a.e. }}{\longrightarrow} f[v] \Longrightarrow f_{n} \stackrel{\text { a.e. }}{\longrightarrow} f[\lambda]
$$

if and only if $\lambda \ll_{I} v$;

(2) for all $f \in \mathcal{F}$ and all $\left\{f_{n}\right\}_{n \in \mathbb{N}} \subset \mathcal{F}$,

$$
f_{n} \stackrel{v}{\longrightarrow} f \Longrightarrow f_{n} \stackrel{\lambda}{\longrightarrow} f
$$

if and only if $\lambda \ll_{V I} v$. 
Proof. From Definition 1 it is easy to obtain the conclusions.

Theorem 2. Let $(\lambda, v) \in \mathfrak{F M} \times \mathfrak{F M}$. Then the following are equivalent:

(1) $\lambda \ll{ }_{I}^{(s)} v$;

(2) for all $f, g \in \mathcal{F}$ and all $\left\{f_{n}\right\}_{n \in \mathbb{N}} \subset \mathcal{F},\left\{g_{n}\right\}_{n \in \mathbb{N}} \subset \mathcal{F}$, we have

$$
f_{n} \stackrel{\text { a.e. }}{\longrightarrow} f[v] \text { and } g_{n} \stackrel{\text { a.e. }}{\longrightarrow} g[v] \Longrightarrow f_{n} \vee g_{n} \stackrel{\text { a.e. }}{\longrightarrow} f \vee g[\lambda] \text {; }
$$

(3) for all $f, g \in \mathcal{F}$ and all $\left\{f_{n}\right\}_{n \in \mathbb{N}} \subset \mathcal{F},\left\{g_{n}\right\}_{n \in \mathbb{N}} \subset \mathcal{F}$, we have

$$
f_{n} \stackrel{\text { a.e. }}{\longrightarrow} f[v] \text { and } g_{n} \stackrel{\text { a.e. }}{\longrightarrow} g[v] \Longrightarrow f_{n}+g_{n} \stackrel{\text { a.e. }}{\longrightarrow} f+g[\lambda] \text {. }
$$

Proof. $(1) \Rightarrow(2)$. If $f_{n} \stackrel{\text { a.e }}{\longrightarrow} f[v]$ and $g_{n} \stackrel{\text { a.e }}{\longrightarrow} g[v]$, then there exist $F, G \in \mathcal{A}$ with $v(F)=v(G)=0$ such that $f_{n} \rightarrow f$ on $X \backslash F$ and $g_{n} \rightarrow g$ on $X \backslash G$. Since $\lambda \ll_{I}^{(s)} v$, we have $\lambda(F \cup G)=0$. Noting that $f_{n} \vee g_{n} \rightarrow f \vee g$ on $X \backslash F \cup G$, therefore $f_{n} \vee g_{n} \stackrel{\text { a.e. }}{\longrightarrow} f \vee g[\lambda]$.

$(2) \Rightarrow(1)$. Let $P, Q \in \mathcal{A}$ and $v(P)=v(Q)=0$. Denote $f_{n}=\chi_{P}$ and $g_{n}=\chi_{Q}$, where $\chi_{P}$ and $\chi_{Q}$ are the characteristic functions of $P$ and $Q$, respectively. It follows from $f_{n} \stackrel{a . e}{\longrightarrow} 0[v]$ and $g_{n} \stackrel{\text { a.e }}{\longrightarrow} 0[v]$ that $f_{n} \vee g_{n} \stackrel{\text { a.e. }}{\longrightarrow} 0[\lambda]$. So $\lambda(P \cup Q)=\lambda\left(\left\{x \in X \mid f_{n}(x) \vee g_{n}(x) \nrightarrow 0\right\}\right)=0$.

$(1) \Leftrightarrow(3)$. The proof is similar.

Theorem 3. Let $(\lambda, v) \in \mathfrak{F M} \times \mathfrak{F M}$. Then the following are equivalent:

(1) $\lambda \ll \ll_{V I}^{(s)} v$;

(2) for all $f, g \in \mathcal{F}$ and all $\left\{f_{n}\right\}_{n \in \mathbb{N}} \subset \mathcal{F},\left\{g_{n}\right\}_{n \in \mathbb{N}} \subset \mathcal{F}$, we have

$$
f_{n} \stackrel{v}{\longrightarrow} f \text { and } g_{n} \stackrel{v}{\longrightarrow} g \Longrightarrow f_{n}+g_{n} \stackrel{\lambda}{\longrightarrow} f+g ;
$$

(3) for all $f, g \in \mathcal{F}$ and all $\left\{f_{n}\right\}_{n \in \mathbb{N}} \subset \mathcal{F},\left\{g_{n}\right\}_{n \in \mathbb{N}} \subset \mathcal{F}$, we have

$$
f_{n} \stackrel{v}{\longrightarrow} f \text { and } g_{n} \stackrel{v}{\longrightarrow} g \Longrightarrow f_{n} \vee g_{n} \stackrel{\lambda}{\longrightarrow} f \vee g
$$

Proof. $(1) \Rightarrow(2)$. Suppose that $f_{n} \stackrel{v}{\longrightarrow} f$ and $g_{n} \stackrel{v}{\longrightarrow} g$. For any given $\epsilon>0$, we denote

$$
F_{n}(\epsilon)=\left\{x \in X|| f_{n}(x)-f(x) \mid \geq \frac{\epsilon}{2}\right\}
$$

and

$$
G_{n}(\epsilon)=\left\{x \in X|| g_{n}(x)-g(x) \mid \geq \frac{\epsilon}{2}\right\}
$$

Then

$$
\left\{x \in X||\left(f_{n}(x)+g_{n}(x)\right)-(f(x)+g(x)) \mid \geq \epsilon\right\} \subset F_{n}(\epsilon) \cup G_{n}(\epsilon) .
$$

Since $f_{n} \stackrel{v}{\longrightarrow} f$ and $f_{n} \stackrel{v}{\longrightarrow} g$, we have

$$
v\left(F_{n}(\epsilon)\right) \bigvee v\left(G_{n}(\epsilon)\right) \longrightarrow 0(n \rightarrow \infty)
$$

Therefore, from $\lambda \ll{ }_{V I}^{(s)} v$, we have

$$
\lambda\left(F_{n}(\epsilon) \cup G_{n}(\epsilon)\right) \longrightarrow 0(n \rightarrow \infty)
$$

Hence it is clear that

$$
\lambda\left(\left\{x \in X||\left(f_{n}(x)+g_{n}(x)\right)-(f(x)+g(x)) \mid \geq \epsilon\right\}\right) \longrightarrow 0(n \rightarrow \infty) .
$$


This shows that $f_{n}+g_{n} \stackrel{\lambda}{\longrightarrow} f+g$.

(2) $\Rightarrow(3)$. If $f_{n} \stackrel{v}{\longrightarrow} f$ and $g_{n} \stackrel{v}{\longrightarrow} g$, then $f_{n}+g_{n} \stackrel{\lambda}{\longrightarrow} f+g$.

Noting that $f_{n}(x) \vee g_{n}(x) \leq f_{n}(x)+g_{n}(x)$, for any $\epsilon>0$ we have

$$
\left\{x \in X|| f_{n}(x) \vee g_{n}(x) \mid \geq \epsilon\right\} \subset\left\{x \in X|| f_{n}(x)+g_{n}(x) \mid \geq \epsilon\right\} .
$$

So

$$
\lim _{n \rightarrow+\infty} \lambda\left(\left\{x \in X|| f_{n}(x) \vee g_{n}(x) \mid \geq \epsilon\right\}\right) \leq \lim _{n \rightarrow+\infty} \lambda\left(\left\{x \in X|| f_{n}(x)+g_{n}(x) \mid \geq \epsilon\right\}\right)=0 .
$$

This shows $f_{n} \vee g_{n} \stackrel{\lambda}{\longrightarrow} 0$.

(3) $\Rightarrow$ (1). For any $\left\{P_{n}\right\}_{n \in \mathbb{N}} \subset \mathcal{A},\left\{Q_{n}\right\}_{n \in \mathbb{N}} \subset \mathcal{A}$ with $\lim _{n \rightarrow \infty} \mu\left(P_{n}\right) \vee \mu\left(Q_{n}\right)=0$, we define measurable function sequences $\left\{f_{n}\right\}_{n \in \mathbb{N}} \subset \mathcal{F}$ and $\left\{g_{n}\right\}_{n \in \mathbb{N}} \subset \mathcal{F}$ by

$$
f_{n}(x)=\chi_{P_{n}}(x)= \begin{cases}0 & \text { if } x \notin P_{n} \\ 1 & \text { if } x \in P_{n}\end{cases}
$$

and

$$
g_{n}(x)=\chi_{Q_{n}}(x)= \begin{cases}0 & \text { if } x \notin Q_{n} \\ 1 & \text { if } x \in Q_{n}\end{cases}
$$

$\mathrm{n}=1,2, \ldots$, then $f_{n} \stackrel{v}{\longrightarrow} 0$ and $g_{n} \stackrel{v}{\longrightarrow} 0$. Thus, $f_{n} \vee g_{n} \stackrel{\lambda}{\longrightarrow} 0$. Therefore for $\epsilon=\frac{1}{2}$, we have

$$
\lim _{n \rightarrow+\infty} \lambda\left(\left\{x \in X \mid f_{n}(x) \vee g_{n}(x) \geq \frac{1}{2}\right\}\right)=0 .
$$

Noting $f_{n} \vee g_{n}=\chi_{P_{n}} \vee \chi_{Q_{n}}=\chi_{P_{n} \cup Q_{n}}$, we have

$$
\left\{x \in X \mid f_{n}(x) \vee g_{n}(x) \geq \frac{1}{2}\right\}=\left\{x \in X \mid \chi_{P_{n} \cup Q_{n}}(x) \geq \frac{1}{2}\right\}=P_{n} \cup Q_{n} .
$$

So

$$
\lim _{n \rightarrow+\infty} \lambda\left(P_{n} \cup Q_{n}\right)=0
$$

Thus we have $\lambda \ll_{V I}^{(s)} v$.

When we take $(\lambda, v)=(\mu, \mu),(\bar{\mu}, \bar{\mu}),(\bar{\mu}, \mu)$, and $(\mu, \bar{\mu})$, respectively, and combine Proposition 1 , then the previous results obtained in [3] (Li et al.) are recovered by Theorems 2 and 3, respectively.

Corollary 1. ([3], Theorem 1) Let $\mu \in \mathfrak{F M}$. Then,

(1) $\mu \ll_{I}^{(s)} \mu$ (i.e., $\mu$ is weakly null-additive) if and only if for any $f, g, f_{n}, g_{n} \in \mathcal{F}, f_{n} \stackrel{\text { a.e }}{\longrightarrow} f[\mu]$ and $g_{n} \stackrel{\text { a.e }}{\longrightarrow}$ $g[\mu] \Longrightarrow f_{n}+g_{n} \stackrel{a \cdot e}{\longrightarrow} f+g[\mu]$.

(2) $\bar{\mu} \ll_{I}^{(s)} \bar{\mu}$ (i.e., $\bar{\mu}$ is weakly null-additive) if and only if for any $f, g, f_{n}, g_{n} \in \mathcal{F}, f_{n} \stackrel{\text { p.a.e }}{\longrightarrow} f[\mu]$ and $g_{n} \stackrel{\text { p.a.e }}{\longrightarrow}$ $g[\mu] \Longrightarrow f_{n}+g_{n} \stackrel{\text { p.a.e }}{\longrightarrow} f+g[\mu]$.

(3) $\bar{\mu} \ll_{I}^{(s)} \mu$ if and only if for any $f, g, f_{n}, g_{n} \in \mathcal{F}, f_{n} \stackrel{\text { a.e }}{\longrightarrow} f[\mu]$ and $g_{n} \stackrel{a . e}{\longrightarrow} g[\mu] \Longrightarrow f_{n}+g_{n} \stackrel{\text { p.a.e }}{\longrightarrow}$ $f+g[\mu]$.

(4) $\mu \ll_{I}^{(s)} \bar{\mu}$ if and only if for any $f, g, f_{n}, g_{n} \in \mathcal{F}, f_{n} \stackrel{\text { p.a.e }}{\longrightarrow} f[\mu]$ and $g_{n} \stackrel{\text { p.a.e }}{\longrightarrow} g[\mu] \Longrightarrow f_{n}+g_{n} \stackrel{\text { a.e }}{\longrightarrow}$ $f+g[\mu]$.

Corollary 2. ([3], Theorem 2) Let $\mu \in \mathfrak{F M}$. Then,

(1) $\mu \ll_{V I}^{(s)} \mu$ (i.e., $\mu$ has (p.g.p.)) if and only if for any $f, g, f_{n}, g_{n} \in \mathcal{F}, f_{n} \stackrel{\mu}{\longrightarrow} f$ and $g_{n} \stackrel{\mu}{\longrightarrow} g \Longrightarrow$ $f_{n}+g_{n} \stackrel{\mu}{\longrightarrow} f+g$. 
(2) $\bar{\mu} \ll_{V I}^{(s)} \bar{\mu}$ (i.e., $\bar{\mu}$ has (p.g.p.)) if and only if for any $f, g, f_{n}, g_{n} \in \mathcal{F}, f_{n} \stackrel{p . \mu}{\longrightarrow} f$ and $g_{n} \stackrel{p \cdot \mu}{\longrightarrow} g \Longrightarrow$ $f_{n}+g_{n} \stackrel{p \cdot \mu}{\longrightarrow} f+g$.

(3) $\bar{\mu} \ll_{V I}^{(s)} \mu$ if and only if for any $f, g, f_{n}, g_{n} \in \mathcal{F}, f_{n} \stackrel{\mu}{\longrightarrow} f$ and $g_{n} \stackrel{\mu}{\longrightarrow} g \Longrightarrow f_{n}+g_{n} \stackrel{p \cdot \mu}{\longrightarrow} f+g$.

(4) $\mu \ll_{V I}^{(s)} \bar{\mu}$ if and only if for any $f, g, f_{n}, g_{n} \in \mathcal{F}, f_{n} \stackrel{p \cdot \mu}{\longrightarrow} f$ and $g_{n} \stackrel{p \cdot \mu}{\longrightarrow} g \Longrightarrow f_{n}+g_{n} \stackrel{\mu}{\longrightarrow} f+g$.

In the following we discuss fuzzy measures defined by fuzzy integral.

Given $v \in \mathfrak{F M}$ and $h \in \mathcal{F}$. Then the Sugeno integral of $h$ with respect to $v$ determines a new fuzzy measure $\lambda_{h} \in \mathfrak{F M}$, as follows:

$$
\lambda_{h}(A)=(S) \int_{A} h d v, \quad \forall A \in \mathcal{A}
$$

Proposition 3. (1) $\lambda_{h} \ll_{I} v$, and $\lambda_{h} \ll_{V I} v$; (2) $\lambda_{h} \ll_{I}^{(s)} v$; (3) If $v$ is continuous from below, then $\lambda_{h} \ll(s) v$.

As a direct result of Proposition 3 and Theorems 2 and 3, we have the following corollary.

Corollary 3. Let $v \in \mathfrak{F M}$. Then,

(1) for all $f, g \in \mathcal{F}$ and all $\left\{f_{n}\right\}_{n \in \mathbb{N}} \subset \mathcal{F},\left\{g_{n}\right\}_{n \in \mathbb{N}} \subset \mathcal{F}$, we have

$$
f_{n} \stackrel{\text { a.e. }}{\longrightarrow} f[v] \text { and } g_{n} \stackrel{\text { a.e. }}{\longrightarrow} g[v] \Longrightarrow f_{n} \vee g_{n} \stackrel{\text { a.e. }}{\longrightarrow} f \vee g\left[\lambda_{h}\right] \text {; }
$$

(2) for all $f, g \in \mathcal{F}$ and all $\left\{f_{n}\right\}_{n \in \mathbb{N}} \subset \mathcal{F},\left\{g_{n}\right\}_{n \in \mathbb{N}} \subset \mathcal{F}$, we have

$$
f_{n} \stackrel{\text { a.e. }}{\longrightarrow} f[v] \text { and } g_{n} \stackrel{\text { a.e. }}{\longrightarrow} g[v] \Longrightarrow f_{n}+g_{n} \stackrel{\text { a.e. }}{\longrightarrow} f+g\left[\lambda_{h}\right] \text {; }
$$

Furthermore, if $v$ is continuous from below, then

(3) for all $f, g \in \mathcal{F}$ and all $\left\{f_{n}\right\}_{n \in \mathbb{N}} \subset \mathcal{F},\left\{g_{n}\right\}_{n \in \mathbb{N}} \subset \mathcal{F}$, we have

$$
f_{n} \stackrel{v}{\longrightarrow} f \text { and } g_{n} \stackrel{v}{\longrightarrow} g \Longrightarrow f_{n} \vee g_{n} \stackrel{\lambda_{h}}{\longrightarrow} f \vee g ;
$$

(4) for all $f, g \in \mathcal{F}$ and all $\left\{f_{n}\right\}_{n \in \mathbb{N}} \subset \mathcal{F},\left\{g_{n}\right\}_{n \in \mathbb{N}} \subset \mathcal{F}$, we have

$$
f_{n} \stackrel{v}{\longrightarrow} f \text { and } g_{n} \stackrel{v}{\longrightarrow} g \Longrightarrow f_{n}+g_{n} \stackrel{\lambda_{h}}{\longrightarrow} f+g .
$$

\section{Conclusions}

We have shown the equivalences between the convergence (a.e. or in measure) of the sum of two convergent sequences of measurable functions and several types of absolute continuity of fuzzy measures. The main results are Theorems 1-3. The characteristics of strong absolute continuity of Type I and Type VI of fuzzy measures have been described by using convergence of sequence of measurable functions. As we have seen, such descriptions were done in a more general context concerning a pair of monotone measures, the previous related results $[3,15]$ become to be some special cases of our new results.

In our discussions we only involved theoretical methods, not presenting a specific application instance. In further research, we will focus on the application of these methods. For instance, we will generalize convergence in measure theorems of nonlinear integrals $[17,18]$ in the general framework concerning a pair of monotone measures, and discuss the linearity of pan-integrals and concave integrals $[19,20]$, our results will be useful.

Funding: This work was supported by the National Natural Science Foundation of China (Grants No. 11571106 and No. 11371332), and the Fundamental Research Funds for the Central Universities.

Acknowledgments: The author is grateful to the referees for their valuable comments for improvements. 
Conflicts of Interest: The author declares no conflict of interest.

\section{References}

1. Berberian, S.K. Measure and Integration; Macmillan: New York, NY, USA, 1965.

2. Halmos, P.R. Measure Theory; Van Nostrand: New York, NY, USA, 1968.

3. Li, J.; Mesiar, R.; Zhang, Q. Absolute continuity of monotone measure and convergence in measure. Commun. Comput. Inf. Sci. (CCIS)) 2010, 80, 500-504.

4. Li, J.; Yasuda, M.; Jiang, Q.; Suzuki, H.; Wang, Z.; Klir, G.J. Convergence of sequence of measurable functions on fuzzy measure space. Fuzzy Sets Syst. 1997, 87, 317-323. [CrossRef]

5. Li, J.; Mesiar, R.; Pap, E.; Klement, E.P. Convergence theorems for monotone measures. Fuzzy Sets Syst. 2015, 281, 103-127. [CrossRef]

6. Sugeno, M. Theory of Fuzzy Integrals and Its Applications. Ph.D. Dissertation, Takyo Institute of Technology, Takyo, Japan, 1974.

7. Torra, V.; Narukawa, Y.; Sugeno, M. (Eds.) Non-Additive Measure-Theory and Applications; Stud. Fuzziness Soft Comput.; Spinger: New York, NY, USA, 2014; Volume 310.

8. Choquet, G. Theory of capacities. Ann. Inst. Fourier 1953, 5, 131-295. [CrossRef]

9. Kawabe, J. Continuity and compactness of the indirect product of two non-additive measures. Fuzzy Sets Syst. 2009, 160, 1327-1333. [CrossRef]

10. Pap, E. Null-Additive Set Functions; Kluwer: Dordrecht, The Netherlands, 1995.

11. Wang, Z.; Klir, G.J. Generalized Measure Theory; Springer: New York, NY, USA, 2009.

12. Klement, E.P.; Li, J.; Mesiar, R.; Pap, E. Integrals based on monotone set functions. Fuzzy Sets Syst. 2015, 281, 88-102. [CrossRef]

13. Mesiar, R.; Stupňanová, A. Decomposition integrals. Int. J. Approx. Reason. 2013, 54, 1252-1259. [CrossRef]

14. Wang, Z.; Klir, G.J. Fuzzy measures defined by fuzzy integral and their absolute continuity. J. Math. Anal. Appl. 1996, 203, 150-165. [CrossRef]

15. Li, J.; Mesiar, M.; Wu, H. On weak null-additivity of monotone measures. Commun. Comput. Inf. Sci. (CCIS) 2012, 300, 278-285.

16. Dobrakov, I.; Farkova, J. On submeasures II. Math. Slovaca 1980, 30, 65-81.

17. Kawabe, J. Convergence in measure theorems of nonlinear integrals of functions integrable to the pth power. Fuzzy Sets Syst. 2019. [CrossRef]

18. Kawabe, J. The Vitali convergence in measure theorem of nonlinear integrals. Fuzzy Sets Syst. 2020, 379, 63-81. [CrossRef]

19. Ouyang, Y.; Li, J.; Mesiar, R. Coincidences of the concave integral and the pan-integral. Symmetry 2017, 9, 90. [CrossRef]

20. Ouyang, Y.; Li, J.; Mesiar, R. On linearity of pan-integral and pan-integrable functions space. Int. J. Approx. Reason. 2017, 90, 307-318. [CrossRef]

(C) 2020 by the author. Licensee MDPI, Basel, Switzerland. This article is an open access article distributed under the terms and conditions of the Creative Commons Attribution (CC BY) license (http://creativecommons.org/licenses/by/4.0/). 